\title{
Workplace Violence in Social Care Settings: Traumatic Stress and Burnout in Staff
}

\author{
Daniel Dowling \\ Postgraduate \\ Department of Psychology, Dublin Business School \\ Dublin, Ireland \\ Dr. Prakashini Banka \\ School of Nursing and Midwifery, Trinity College Dublin \\ Dublin, Ireland
}

(C) Daniel Dowling and Prakashini Banka. This work is licensed under the Creative Commons Attribution-NonCommercial-ShareAlike 4.0 International License. To view a copy of this license, visit https://creativecommons.org/licenses/by-nc-sa/4.0/ .

\section{Abstract}

The aim of this study was to examine the relationship between frequency of exposure to physical violence/aggression and traumatic stress and burnout in social care workers (SCWs). One hundred SCWs were surveyed using non-experimental, cross-sectional, quantitative correlational study design with three questionnaires: Demographic Questionnaire, Revised Impact of Events Scale and Maslach Burnout Inventory. Responses were analysed using SPSS quantitative software and utilised descriptive statistics, and non-parametric correlational tests. Results indicated statistically significant positive relationships between the frequency of exposure to physical aggression/violence and traumatic stress and burnout measures. Results also indicated significant positive correlations between traumatic stress and burnout. Furthermore, exposure to workplace violence/aggression, traumatic stress, and burnout also positively correlated with SCWs desire to leave the profession in the next five years. Finally, results indicated a significant relationship between frequency of self-care and levels of traumatic stress and burnout subscales in SCWs.)

Keywords: Workplace Violence; Traumatic Stress; Burnout; Social Care Workers.

\section{Introduction}

\section{The Current Landscape of Social Care Work in Ireland}

Social care workers (SCWs) and Assistant Support Workers (ASW) plan and provide front-line care to vulnerable adults and children who experience disability, disadvantage, and marginalisation in a variety of settings. The role of SCWs and ASWs involves advocating, caring for, and supporting individuals to achieve their full potential (Lalor and Share, 2013). However, there are many physical and psychological challenges which SCWs often encounter daily which may have a profound impact on the quality of life of these primary care givers. The extent of these challenges is only coming to light in recent years.

Social Care Ireland published the Crisis, Concern and Complacency report in 2016 (Keogh and Byrne, 2016), which found that $90 \%$ of SCWs in Ireland have experienced 
regular abuse, threats, and physical violence in the workplace, while $100 \%$ of SCWs working in children's residential services experienced workplace violence (Keogh and Byrne, 2016). The authors also suggest there appears to exist a culture of complacency and normality/desensitisation to workplace violence at present. This culture was found to extend up to management and agency level (Keogh and Byrne, 2016). Many other studies have highlighted the risk of workplace violence SCWs are exposed to and this risk is increasing (Alink et al., 2014; Colton and Roberts, 2006; Franz, 2010; Harris and Leather, 2012; McAdams, 2002). The Health and Safety Authority defines workplace violence and aggression as 'any incident where staff are abused, threatened or assaulted in circumstances related to their work' (Health and Safety Authority, 2014). The Economic and Social Research Institute (ESRI) found that demand for public and private health and social care services in Ireland will increase exponentially by 2030 (Wren et al., 2017). These changes will have significant and profound impacts on the health and social care sector which is already characterised by challenges of staff retention (Keogh and Byrne, 2016; Barak, Nissly and Levin, 2001).

The type of care setting is also important in terms of workplace violence, for example, SCWs in disability services have been found to be exposed to high levels of workplace violence (Hensel, Lunsky and Dewa, 2012), while those working in settings such as family support and community services may be at a lower risk. Staff retention rates seem to differ depending on the care setting and this may suggest that exposure to workplace violence may have a direct link to SCW retention rates (Keogh and Byrne, 2016). Residential care in particular can be a complex and unpredictable environment characterised by the spectrum of diagnoses and unique personal circumstances of each service user, combined with difficulties associated with communal living, all of which can have considerable effects on service user's behaviour (Clough, Bullock and Ward, 2006). Furthermore, young people in residential care may have experienced disproportionately high levels of social disadvantage and trauma prior to entering residential social care that may also influence their behaviour towards care givers (Brodie, 2005). Howard (2014) stresses that residential care can be chaotic, ambivalent, turbulent, unpredictable, and often dangerous for staff and young people (Howard, 2014).

\section{Literature Review}

\section{The Impact of Workplace Violence}

Routine exposure to workplace violence can have significant deleterious effects on quality of life for SCWs/ASWs and indeed the service users they care for. Studies have found that incidents of aggression/violence can result in primary, secondary and tertiary victims including service users who may experience traumatic stress and anxiety (Hastings and Brown, 2002; Rippon, 2000). SCWs may experience feelings of despair, sadness, annoyance, anger and anxiety when faced with violent incidents (Emerson and Hatton, 2000). Growing evidence is highlighting the extent to which SCWs/ASWs are exposed to workplace violence and the potential consequences. However, the full ramifications of this working environment are still lacking. Furthermore, the Crisis, Concern and Complacency report highlighted the fact that 
very little attention has been paid to this situation by policy makers since the first report in 2001 (Keogh et al., 2001; Keogh and Byrne, 2016).

The personal cost of experiencing violence include the immediate physical injuries, some of which may be significant, but may also cause various immediate and longterm psychological effects and a complex range of emotions (Lovell and Skellern, 2013). Fear, stress, frustration, guilt, anger, annoyance, and anxiety have been identified as common impacts on SCWs and social workers who experience violence (Keogh and Byrne, 2016; Littlechild, 2000; Smith and Nursten, 1998). Fear responses linked to direct and vicarious exposure to violence were found to be related to subsequent depression, anxiety and had a negative impact on the well-being of SCWs (Schat and Kelloway, 2000). These emotional states may cause distress to SCWs and may lead to feelings of being unsafe, sleeplessness, anger, and irritability (Keogh and Byrne, 2016). The multifactorial nature of SCW can also place emotional demands on staff, and staff may benefit from regular support groups and supportive supervision (Taylor, 2011). These emotional and psychological effects are not only felt after violent incidents but may also be felt prior to violent incidents, whereby SCWs may experience anticipatory anxiety by predicting/anticipating incidents in advance. Some SCWs reported feeling relieved after behavioural outburst had occurred which may remove the tension and fear of anticipated violence (Keogh and Byrne, 2016). Other sentiments that SCWs reported feeling after a violent incident included feelings of failure, disillusionment, disappointment, powerlessness, and embarrassment (Keogh and Byrne, 2016). More alarming are statistics disclosed by the Office for National Statistics (2017), in its report 'Suicide by Occupation, England: 2011 to 2015'. Analysis found that care workers of both genders were at risk of suicide at almost twice the national average (Office for National Statistics, 2017).

While emotional and psychological issues may arise for many SCWs in response to exposure to violence/aggression, others may become desensitised and view incidents as a 'normal' part of working life (Lundström, Åström, and Graneheim, 2007), and while not acceptable, violence may be considered as tolerable in some care settings (Lovell and Skellern, 2013). The World Health Organization (WHO) identified social and cultural norms that are seen as rules or expectations of behaviour within certain groups and can become the standard of acceptable or unacceptable behaviour. These expectations of behavioural norms within certain groups can even encourage violence and aggression (Keogh and Byrne, 2016; World Health Organization, 2009). As many SCWs perceived violence as part of the job (Keogh et al., 2001), incidents of workplace violence have been significantly underreported (McKenna, 2004). This may be due to a belief that nothing would be done if they reported it, potentially as a result of previous reports not being acted on. Staff may also be fearful of being perceived as unskilled, not up to the job, or could be experiencing the strain of the role and may not have time to fill out incident reports (Keogh et al., 2001). Colton and Roberts (2007) identified the need for organisations to clearly convey to all individuals participating in care settings that workplace violence is unacceptable by ensuring there are sound, researchinformed strategies to prevent, manage and support staff after violent incidents (Colton and Roberts, 2007). As many organisations do not currently have adequate support structures in place for staff, this can lead to high levels of stress and may ultimately be 
a significant contributory factor in staff burnout and also SCWs perceiving their profession as a stepping stone to other employment areas (Colton and Roberts, 2007).

\section{Traumatic Stress and Burnout}

The most reported personal impact of workplace violence in SCWs was stress with $98 \%$ of SCWs surveyed reporting this effect (Keogh and Byrne, 2016). In social care settings, regular verbal and physical threats of violence and aggression can have an impact on SCWs stress levels and may even result in post-traumatic stress disorder (PTSD) in some individuals and this may impact staff retention (Balloch, Pahl and McLean, 1998; Colton and Roberts, 2006; Colton and Roberts, 2007; Hastings and Brown, 2002; Keogh and Byrne, 2016; Santos et al., 2009; Whitaker, Archer and Hicks, 1998). Chronic stress can bring SCWs to the point of burnout and this impacts the individual, the entire care team, and wider social networks (Keogh and Byrne, 2016).

Burnout is a type of psychological stress, sometimes referred to as occupational burnout (Ruotsalainen et al., 2016). Burnout is a syndrome characterised by three types of feelings; emotional exhaustion, depersonalisation, and low personal accomplishment (Maslach et al., 2016). Burnout can negatively impact quality of life, can be personally distressing (Freudenberger, 1975), and can have numerous health consequences (Honkonen et al., 2006). Burnout may also be an important risk factor or precursor to compassion fatigue/secondary traumatic stress (Collins and Long, 2003). Compassion fatigue, like burnout, can challenge SCWs ability to provide adequate care and maintain personal and professional therapeutic relationships. While burnout is a gradual process, compassion fatigue can be sudden and acute (Collins and Long, 2003; Figley 1995). Lloyd, King and Chenoweth (2002) found that social workers may experience higher levels of stress and burnout than comparable occupational groups (Lloyd, King and Chenoweth, 2002). Maslach (2016) predicted that burnout would be related to the desire to leave one's job (Maslach et al., 2016). Healy, Meagher and Cullin (2007) highlighted high levels of staff turnover and retention challenges in the care profession as a result of stress and burnout (Healy, Meagher, and Cullin, 2007).

The relationship between exposure to aggression/violence and burnout has been highlighted in previous studies investigating this link in various professions including: nurses (Galián-Muñoz et al., 2016), nursing home caregivers (Isaksson et al., 2008), police officers (Kop, Euwema, and Schaufeli, 1999), psychiatrists (Kumar, 2007), and social workers (Beaver, 1999).

\section{Rationale, Aims and Hypotheses}

The current research study aimed to investigate whether SCWs/ASWs who have been exposed to workplace violence report a significant level of traumatic stress and burnout symptomology. Furthermore, does the frequency of workplace violence impact the levels of traumatic stress and burnout in SCWs? This study also aims to examine the potential protective role of self-care in moderating the perceived effects of workplace violence/aggression on traumatic stress and burnout in SCWs. 
Hypothesis $1(\mathbf{H} 1)$ : There will be a significant positive relationship between the frequency of exposure to workplace aggression/violence and levels of traumatic stress.

Hypothesis 2 (H2): There will be a significant positive relationship between the frequency of exposure to workplace aggression/violence and burnout symptomology.

Hypothesis $3(\mathbf{H} 3)$ : There will be a significant positive correlation between traumatic stress and burnout.

Hypothesis $4(\mathbf{H} 4)$ : There will be a significant positive correlation between the frequency of exposure to workplace aggression/violence and desire to leave the SCW profession.

Hypothesis 5 (H5): There will be a significant positive correlation between traumatic stress scores and desire to leave the SCW profession.

Hypothesis $6(\mathrm{H6})$ : There will be a significant correlation between Maslach burnout subscales and desire to leave the SCW profession.

Hypothesis $7(\mathbf{H 7})$ : There will be a significant negative correlation between self-care and traumatic stress and burnout.

\section{Methodology}

\section{Participants}

Participants were SCWs and ASWs currently working in front-line care positions in the private sector within Ireland. Participants $(n=100)$ were invited to complete an inperson paper-based survey which took approximately 5-10 minutes. The mean and standard deviation of participants age among other descriptive variables was ascertained. Inclusion criteria for participation required participants to be over 18 and have current or previous experience of workplace violence in social care settings. Participation was voluntary as per section 1.3.7 of the PSI Code of Professional Ethics and was anonymous. No monetary incentives were provided, and participants were informed how their responses would be used and presented. To gain access to participants, prior consent was given by the person in charge of residential units.

\section{Design}

A quantitative correlational study design was employed to investigate relationships between variables. Each participant, recruited through non-probability purposive sampling, completed the same survey. The survey was comprised of a demographic questionnaire, the revised Impact of Events Scale (IES-r) and the Maslach Burnout Inventory - Human Services Survey (MBI-HSS). Predictor variables were: Frequency of exposure to physical aggression/violence, length of service, self-care. Criterion variables were burnout and traumatic stress, measured using the MBI-HSS (Maslach et al., 2016) and the IES-r (Weiss and Marmar, 1997) respectively. 


\section{Materials}

\section{Demographic Questionnaire}

A demographic questionnaire was administered to gather role specific information for the purpose of using as predictor descriptives. Questions asked and responses available included: 'Have you experience physical violence in the workplace' (Yes/No), 'Gender you identify with' (Female/Male), 'Age' (_Years), 'Work Schedule' (Full/Part Time), 'Years of employment as Social Care Worker or Assistant Support Worker' (_Years), 'Current role' (Front line staff/Management), 'Main area of work' (Day service/Residential service/Outreach/Community service/Other), 'How often do you personally experience physical aggression/violence' (Daily/ Weekly/ Monthly/ Yearly/ Never), 'How often do you witness physical aggression/violence against colleagues' (Daily/Weekly/Monthly/Yearly/Never), 'Do you feel like there is a culture of normality towards being exposed to physical violence' (Yes/No), 'Do you feel like being exposed to physical violence is expected of you from your employer' (Yes/No), 'Do you feel like the current supports available to you as a social care professional are adequate to maintain your physical and mental wellbeing' (Yes/No), 'Do you engage in self-care activities, e.g. exercise, mindfulness, making time for yourself etc.' (Daily/Weekly/Monthly/Never), 'Do you see yourself or wish to see yourself in the social care profession in 2-3 years' time' (Yes/No), 'Do you see yourself or wish to see yourself in the social care profession in 5 years' time' (Yes/No).

\section{Impact of Events Scale - Revised Edition}

The IES-R is a self-administered, 22-item questionnaire based on three types of symptoms identified in the Diagnostic and Statistical Manual of Mental Disorders, third edition, as indicators of posttraumatic stress disorder (PTSD) (Spitzer and Williams, 1980). The three types are: Intrusion (INT), Avoidance (AVD) and Hyperarousal (HYP). The IES-R is not a diagnostic or screening tool for PTSD, rather, it relies on self-reporting of symptoms after a traumatic event. Participants are asked to indicate the degree of distress for 22 symptoms according to a five-point scale: 0 indicates the symptom occurs "not at all"; 1, "a little bit"; 2, "moderately"; 3, "quite a bit"; and 4, "extremely" (Weiss and Marmar, 1997). A score of 24 or more may represent a clinical concern for PTSD (Asukai et al., 2002). A score of 33 or more represents the best cut off for probable diagnosis of PTSD (Creamer, Bell and Failla, 2003). A score of 37 or more is high enough to potentially suppress immune system function for as much as ten years after the traumatic event (Kawamura, Kim and Asukai, 2001). The revised Impact of Event Scale is one of the most widely used self-report measures for traumatic stress and has high reliability and validity scores (Beck et al., 2008).

\section{Maslach Burnout Inventory - Human Services Survey (MBI-HSS)}

Maslach Burnout Inventory ( $\mathrm{MBI}$ ) is recognised as the leading measure of burnout and the MBI-HSS is the original and most widely used version of the MBI. It was designed to capture feelings of burnout among people working in human services, where professionals such as SCWs spend considerable time interacting intensely with clients (Maslach et al., 2016). The reliability of the MBI-HSS exceeds the recommended levels for research instruments and the validity has been demonstrated by numerous studies and meta-analytic reviews confirming hypotheses regarding relationships between job characteristics and burnout (Maslach et al., 2016). 
The MBI-HSS analyses three central aspects of burnout: emotional exhaustion (EE), depersonalisation (DP), and lack of personal accomplishment (PA). The frequency by which participants experience feelings related to each scale is assessed using a seven-point scale (Maslach et al., 2016). When interpreting results, it is important to note there is no definitive score proving participants are 'burnt out'. The mean scores are considered where they fall on the 7-point scale from 0 (Never) to 6 (Daily). For example, an EE mean score of 5.5 would indicate the participant felt emotionally exhausted several times a week on average, but not every day. The three MBI-HSS scores should be calculated and interpreted separately and should not be combined to form a single "burnout" score. For ease of interpretation, mean responses were calculated.

\section{Procedure}

This research was carried out between December 2018 and February 2019. The researcher attended several residential and day service units within a healthcare service provider in the midlands region of Ireland. The aim of the research was explained to staff at a time that was suitable for them and then information and consent forms in addition to the survey were provided to staff to complete. Written instructions were in the survey booklet. Any questions participants had were answered at the time. After completion, staff were checked to ensure they were okay. Support services were then outlined and signposted verbally and in the debrief form.

\section{Ethical Considerations}

Ethical approval was granted by Dublin Business School Ethics Board. Throughout this study the PSI Code of Ethics (Psychological Society of Ireland, 2010) was always adhered to.

\section{Data Analysis}

Descriptive and inferential statistics were employed during analysis. Mean scores and standard deviation results were used for demographic variables to highlight raw data. As data was not normally distributed, inferential statistics included Spearman correlations to test for relationships between predictor and criterion variables.

\section{Results}

\section{Descriptive Statistics \\ Demographic Questionnaire Descriptives}

Participants ranged in age from 21 to 54 , with mean age of 32 ( $S D=7.56$ ), with $56 \%$ females, and $44 \%$ males. The mean time employed as SCWs/ASWs was 5 years $(S D=4.55)$, with the shortest serving 6 months and longest 25 years. Seventy-nine percent worked full-time, $21 \%$ worked part-time and $86 \%$ were front-line staff while $14 \%$ were management. Participants worked in day service (10\%), residential service $(87 \%)$, and outreach/community service (3\%).

Age

The mean age (Years) of participants in the Daily (30.25), Weekly (30.77), Monthly (32.49), and Yearly (36) exposure groups was ascertained. The mean age in the 
combined Daily-Weekly group and Monthly-Yearly group was 30.63 years and 33.6 years respectively.

\section{Length of Service}

The mean length of service (Years) in the Daily (3.25), Weekly (4.07), Monthly (5.08), and Yearly (7.83) exposure groups was ascertained. The mean length of service in the combined Daily-Weekly group and Monthly-Yearly group was 3.84 years and 5.95 years respectively and is outlined in Figure 1. This result highlights differences in length of time people have worked in each of the different exposure to aggression/violence groups.

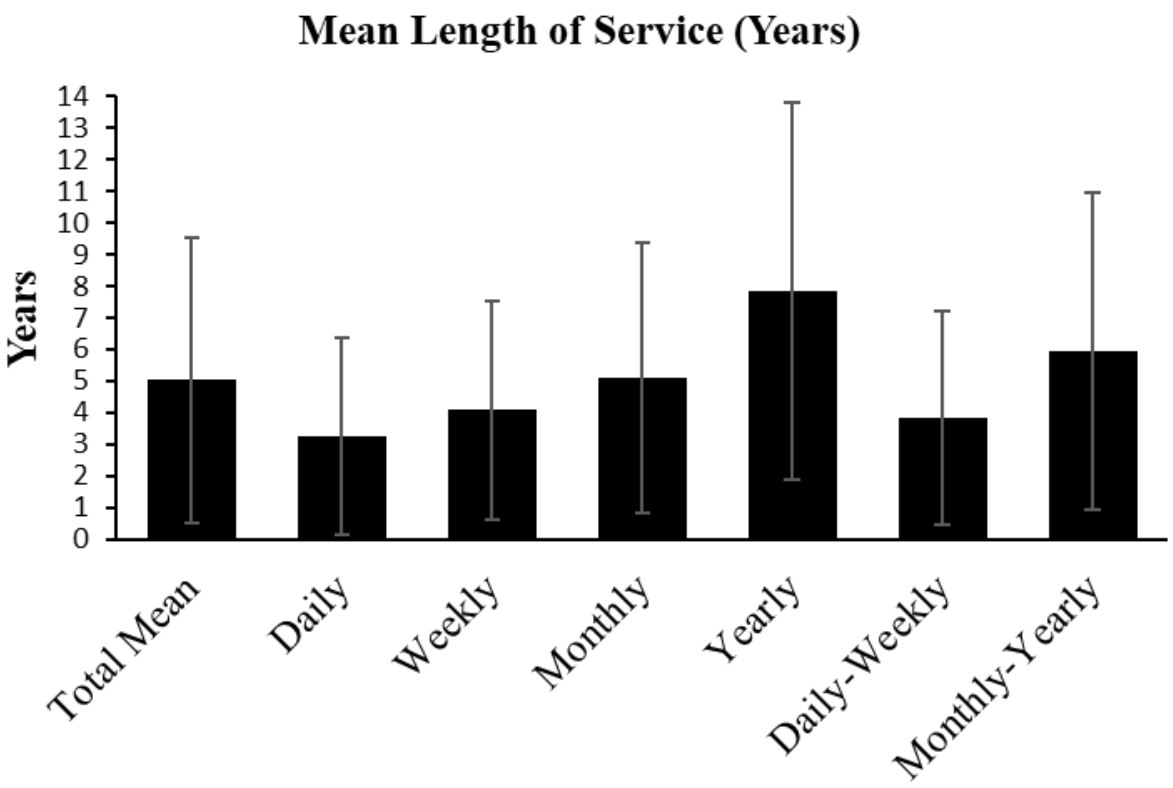

Figure 1: Mean length of service (years) in each frequency of exposure to physical aggression and violence groups. Error bars denote the standard deviation around the means.

\section{Frequency of Exposure to Physical Aggression or Violence}

SCWs/ASWs described being exposed to physical aggression and violence daily $(12 \%)$, Weekly (31\%), Monthly (39\%) and Yearly (18\%). The number of SCWs exposed to physical aggression and violence Daily/Weekly comprised $43 \%$ of the sample and those exposed Monthly/Yearly comprised $57 \%$ of the sample. Interestingly, the mean length of service in each of these categories followed a pattern of decreasing length of service with higher levels of exposure to physical aggression and violence, as outlined in Figure 1.

\section{Culture of Normality}

When participants were asked if they thought there was a culture of normality towards being exposed to physical violence or aggression in the workplace, $92 \%$ indicated there was. This opinion was shared among all exposure groups, front-line staff and management. 


\section{Employer Expectation}

When participants were asked if they felt being exposed to physical violence or aggression is expected of them from their employer, $81 \%$ felt this expectation was present. This opinion was shared among front-line staff and management and each exposure group.

\section{Available Supports}

When participants were asked if they felt current supports available to them were adequate to maintain physical and mental well-being, 33\% indicated they did, while $67 \%$ did not. The majority of the Daily, Weekly, and Monthly exposure groups did not feel that adequate supports were available, while the majority of those in the yearly exposure group felt there were adequate supports available.

\section{$\underline{\text { Self-care }}$}

Participants were asked how frequently they engaged in self-care activities, such as exercising, mindfulness, making time for themselves. Participants engaging in Daily $(36 \%)$, Weekly (41\%), Monthly (18\%), and Never (5\%) categories were ascertained.

\section{Future Career Outlook}

The choice of using a 2-3 year and 5-year career outlook was used to explore participants short-to-medium term career outlooks. In response to the question "Do you see yourself or wish to see yourself in the social care profession in 2-3 years' time", $68 \%$ of participants indicated they would, while $32 \%$ indicated they would not. When broken down into the different frequencies of exposure to aggression, we can see differing future career outlooks for each: Daily (Yes: $50 \%$, No: 50\%), Weekly (Yes: 58\%, No: 42\%), Monthly (Yes: 72\%, No: 28\%), Yearly (Yes: $89 \%$, No: 11\%). When looking at combined groups we see the Daily-Weekly (Yes: 56\%, No: 44\%) and Monthly-Yearly (Yes: 77\%, No: 23\%). See figure 2.

\section{Three Year Career Outlook}

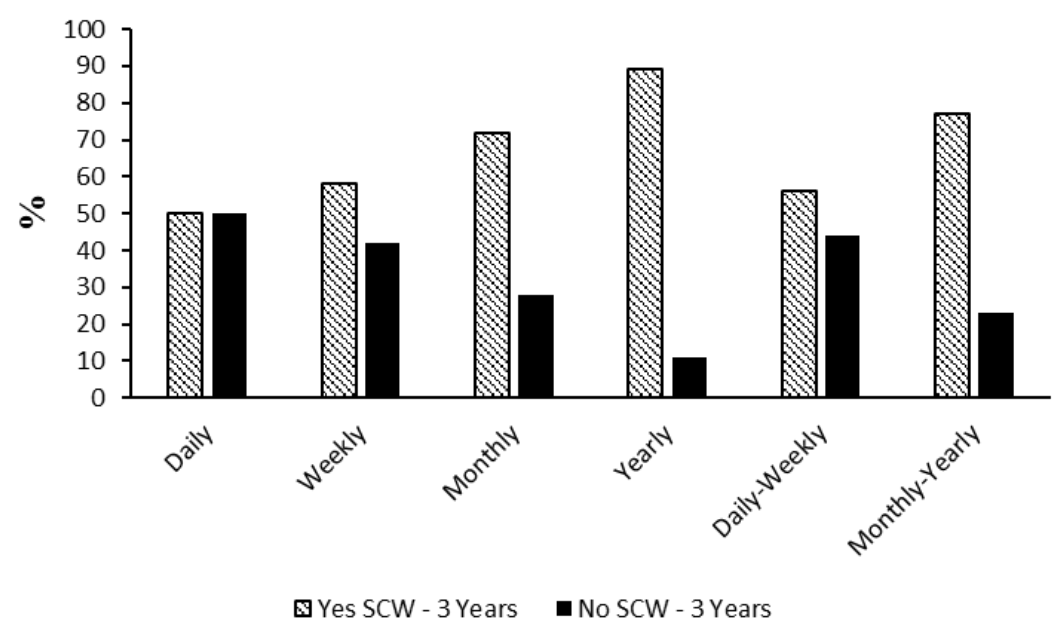

Figure 2: Three-year career outlook for SCWs. 'Yes SCW' indicates percentage who wish to continue in SCW in three years and 'No SCW' indicates percentage who do not wish to continue in SCW in three years. Exposure groups illustrated as Daily, Weekly, Monthly, Yearly and combined groups Daily-Weekly and Monthly-Yearly. 
When asked their 5-year outlook, 57\% indicated they did, while $43 \%$ would not like to be in SCW in 5 years. When broken down into the different frequencies of exposure to aggression groups, we can see differing future career outlooks; Daily (Yes: 42\%, No: 58\%), Weekly (Yes: 32\%, No: 68\%), Monthly (Yes: 67\%, No: 33\%), Yearly (Yes: $89 \%$, No: 11\%). When looking at the combined groups we see the Daily-Weekly (Yes: 35\%, No: 65\%) and the Monthly-Yearly (Yes: 74\%, No: 26\%). See Figure 3.

\section{Five Year Career Outlook}

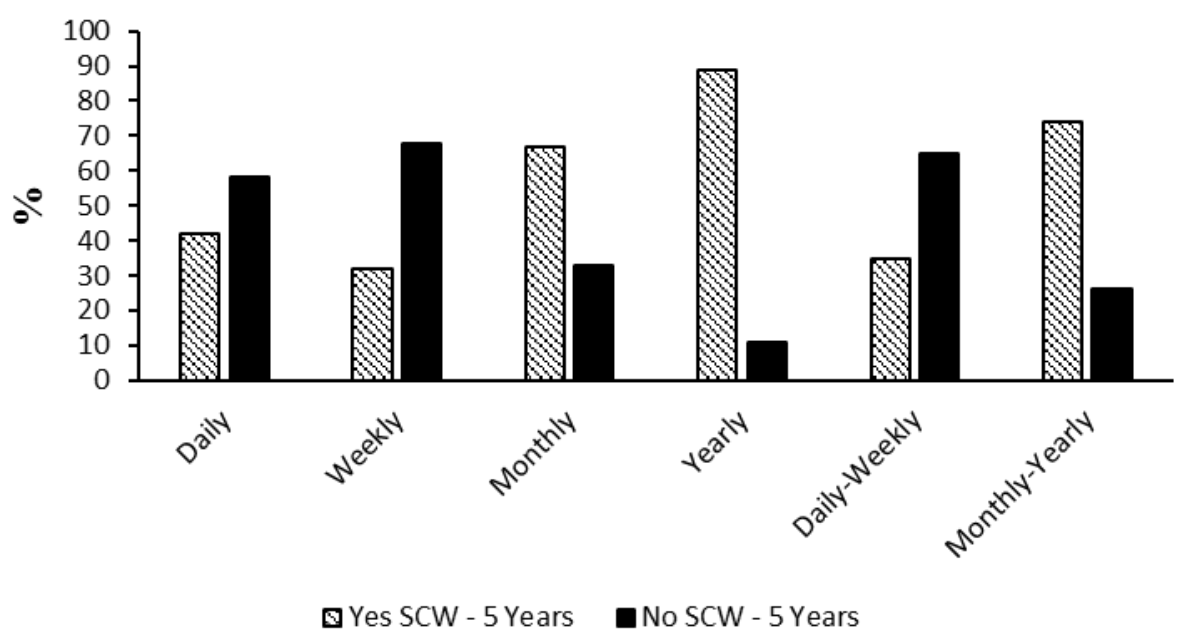

Figure 3: Five-year career outlook for SCWs. 'Yes SCW' indicates percentage who wish to continue in SCW in five years and 'No SCW' indicates percentage who do not wish to continue in SCW in five years. Exposure groups illustrated as Daily, Weekly, Monthly, Yearly and combined groups Daily-Weekly and Monthly-Yearly.

\section{Post-traumatic Stress}

The average total IES-r score for all participants was 22.56, with mean score of 1.02 . To ensure meaningful reporting of results, highlighting relationships between workplace violence and stress/burnout in individuals exposed to different frequencies of physical aggression/violence, each exposure group and combined groups were analysed. Total and mean scores for individual subscales within the IES-r were calculated for all participants and each exposure group. Daily Exposure $(n=12)$ : Total IES-r (43.25) and Total Mean (1.96). Mean subscale scores: Intrusive thoughts (2.02), Avoidance (1.85), Hyperarousal (2.03). Weekly Exposure $(\mathrm{n}=31)$ : Total IES-r (24.61), Total Mean (1.12). Mean subscale scores: Intrusive thoughts (1.23), Avoidance (.9), Hyperarousal (1.23). Monthly Exposure $(\mathrm{n}=39)$ : Total IES-r (19.21), Total Mean (.87). Mean subscale scores: Intrusive thoughts (.88), Avoidance (.91), Hyperarousal (.8). Yearly Exposure $(\mathrm{n}=18)$ : Total IES-r (12.5), Total Mean (.57). Mean subscale scores: Intrusive thoughts (.54), Avoidance (.6), Hyperarousal (.56). Daily-Weekly Exposure Combined $(n=43)$ : Total IES-r (29.81), Total Mean (1.35). Mean subscale scores: Intrusive thoughts (1.45), Avoidance (1.16), Hyperarousal (1.45). Monthly-Yearly Exposure Combined $(\mathrm{n}=57)$ : Total IES-r (17.09), Total Mean (.78). Mean subscale scores: Intrusive thoughts (.77), Avoidance (.81), Hyperarousal (.72). 


\section{Burnout}

Mean scores for individual subscales within the MBI-HSS were calculated for all participants and each exposure group. Total scores $(n=100)$ were: Emotional Exhaustion (EE) (2.74), Depersonalisation (DP) (1.57), Personal Accomplishment (PA) (4.14). Daily Exposure $(\mathrm{n}=12)$ : EE (3.86), DP (2.77), PA (3.6). Weekly Exposure $(n=31)$ : EE (3.27), DP (2.1), PA (3.56). Monthly Exposure $(n=39)$ : EE (2.42), DP (1.08), PA (4.43). Yearly Exposure $(\mathrm{n}=18)$ : EE (1.76), DP (.94), PA (4.89). DailyWeekly Exposure $(\mathrm{n}=43)$ : EE (3.43), DP (2.29), PA (3.57). Monthly-Yearly Exposure $(n=57)$ : EE (2.21), DP (1.04), PA (4.58).

\section{Inferential Statistics}

Preliminary statistical analysis found some data was non-normally distributed. Accordingly, non-parametric tests, were used to analyse all variables. A summary of all correlational analysis can be found in Table 1.

\section{Traumatic Stress $(\mathrm{H} 1)$}

Spearman's Rho correlation coefficients indicated a strong significant positive relationship between frequency of exposure to physical aggression/violence and levels of traumatic stress in all measures of the IES-r except the Avoidance subscale, which had a medium significant positive relationship. IES-Total/mean score $\left(r_{\mathrm{s}}=.527\right.$, $p<.001)$. Mean IES-Intrusion $\left(r_{\mathrm{s}}=.538, p<.001\right)$. Mean IES-Avoidance $\left(r_{\mathrm{s}}=.354, p\right.$ $<.001)$. Mean IES-Hyperarousal $\left(r_{\mathrm{s}}=.517, p<.001\right)$. Combined Daily-Weekly and Monthly-Yearly groups also found a medium significant positive relationship between frequency of exposure to physical aggression/violence and higher levels of traumatic stress in all measures of the IES-r except the Avoidance subscale which had a small significant positive relationship. IES-Total/mean score $\left(r_{\mathrm{s}}=.429, p<.001\right)$. Mean IESIntrusion $\left(r_{\mathrm{s}}=.461, p<.001\right)$. Mean IES-Avoidance $\left(r_{\mathrm{s}}=.240, p=.016\right)$. Mean IESHyperarousal $\left(r_{\mathrm{s}}=.453, p<.001\right)$. Therefore, the null hypothesis was rejected; higher frequency of exposure to physical aggression/violence was related to higher levels of traumatic stress.

\section{Burnout (H2)}

Spearman's Rho correlation coefficients indicted a strong significant positive relationship between higher frequencies of exposure to physical aggression/violence and higher levels of burnout in two of Maslach's burnout inventory subscale; Emotional Exhaustion $\left(r_{\mathrm{s}}=.534, p<.001\right)$, Depersonalisation $\left(r_{\mathrm{s}}=.500, p<.001\right)$, and a medium negative relationship with the Personal Accomplishment subscale $\left(r_{\mathrm{s}}=-.476, p<\right.$ .001). Combined Daily-Weekly and Monthly-Yearly groups also found a medium significant positive relationship between higher frequencies of exposure to physical aggression/violence and higher levels of burnout in two of Maslach's burnout inventory subscale; Emotional Exhaustion $\left(r_{\mathrm{s}}=.481, p<.001\right)$, Depersonalisation $\left(r_{\mathrm{s}}=.498, p\right.$ $<.001$ ), and a medium negative relationship with the Personal Accomplishment subscale $\left(r_{s}=-.469, p<.001\right)$. Therefore, the null hypothesis was rejected; higher frequency of exposure to physical aggression/violence was related to higher levels of staff burnout scores in the emotional exhaustion and depersonalisation subscales and lower levels of personal accomplishment scores. 


\section{Correlation between Traumatic Stress and Burnout (H3)}

Spearman's Rho correlation coefficients indicated a medium significant positive relationship between total mean IES-r (traumatic-stress) and burnout in two of Maslach's burnout inventory subscales; Emotional Exhaustion $\left(r_{\mathrm{s}}=.497, p<.001\right)$, Depersonalisation $\left(r_{\mathrm{s}}=.484, p<.001\right)$, and a medium negative relationship with the Personal Accomplishment subscale $\left(r_{\mathrm{s}}=-.419, p<.001\right)$. Scatter plots were produced as both variables were scale data and can be seen in Figure 4.
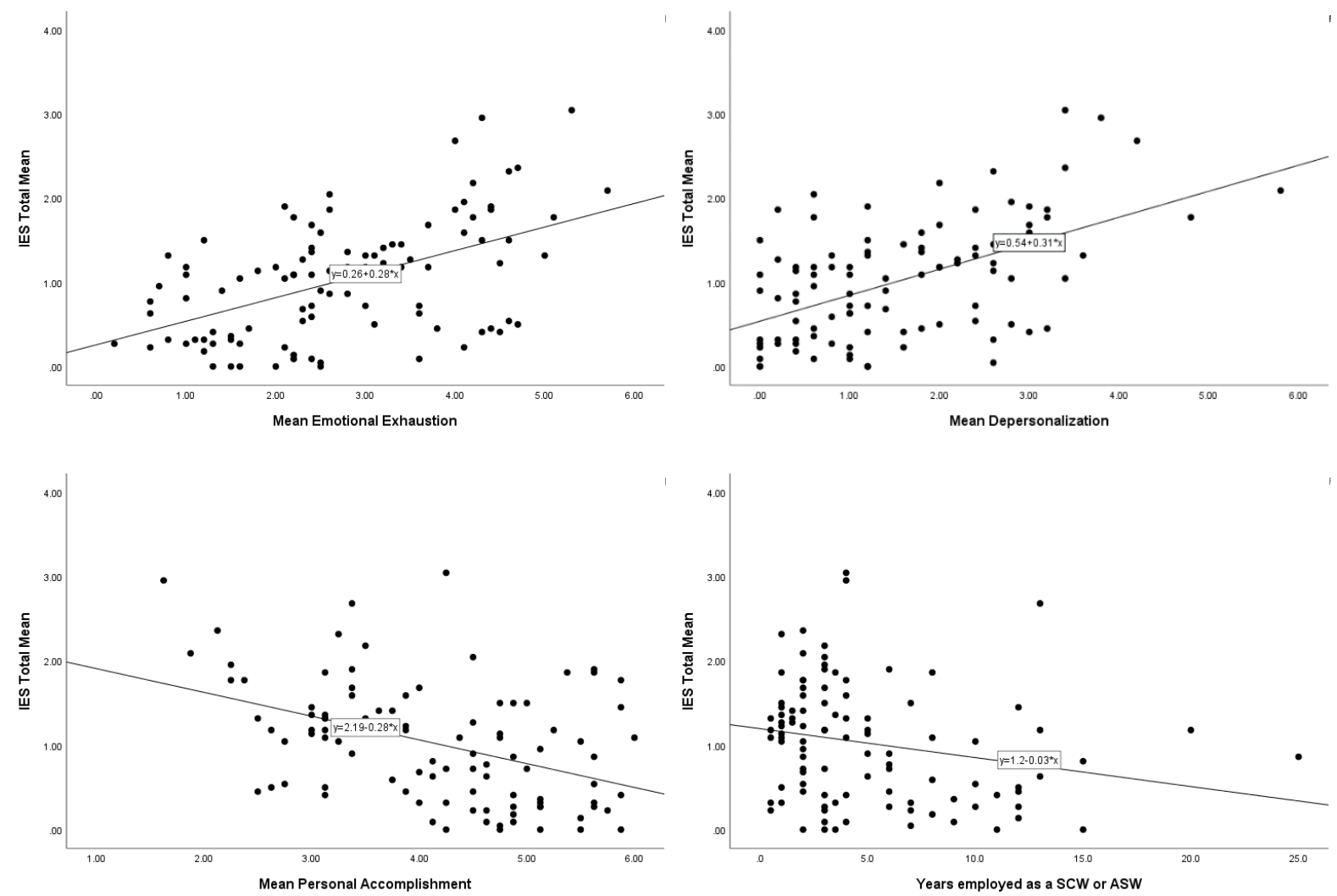

Figure 4: Spearman's Rho correlation between traumatic stress and burnout. Total Mean IES-r was correlated with 'Years employed as a SCW and Maslach Burnout subscales; Emotional Exhaustion, Depersonalisation, Personal Accomplishment.

\section{Impact of Workplace Violence/Aggression, Traumatic Stress, and Burnout on Desire to Leave SCW}

\section{Exposure to Workplace Violence/Aggression and Desire to Leave SCW (H4)}

Spearman's Rho correlation coefficients $(n=100)$ indicated a small positive relationship and a medium positive relationship between frequency of exposure to workplace violence/aggression and desire to leave SCW in the next two-to-three years $\left(r_{\mathrm{s}}=.263, p=.008\right)$ and five years respectively $\left(r_{\mathrm{s}}=.395, p<.001\right)$.

\section{Traumatic Stress and Desire to Leave SCW (H5)}

Spearman's Rho correlation coefficients indicated a small positive relationship and a medium positive relationship between total IES-r traumatic stress scores and desire 
to leave SCW in the next two-to-three years $\left(r_{\mathrm{s}}=.266, p=.007\right)$ and five years respectively $\left(r_{\mathrm{s}}=.305, p=.002\right)$.

\section{Burnout and Desire to Leave SCW (H6)}

Spearman's Rho correlation coefficients indicated a strong positive relationship between Emotional Exhaustion scores and desire to leave SCW in the next two-tothree years $\left(r_{\mathrm{s}}=.516, p<.001\right)$ and five years $\left(r_{\mathrm{s}}=.602, p<.001\right)$.

Spearman's Rho correlation coefficients indicated a medium positive relationship between Depersonalisation scores and desire to leave SCW in the next two-to-three years $\left(r_{\mathrm{s}}=.366, p<.001\right)$ and five years $\left(r_{\mathrm{s}}=.453, p<.001\right)$.

Spearman's Rho correlation coefficients indicated a medium negative relationship between Personal Accomplishment scores and desire to leave SCW in the next twoto-three years $\left(r_{\mathrm{s}}=-.355, p<.001\right)$ and five years $\left(r_{\mathrm{s}}=-.482, p<.001\right)$.

\section{Self-care}

Self-reported frequencies of self-care engagement highlighted individuals who engage in Daily $(n=36)$, Weekly $(n=41)$, Monthly $(n=18)$ self-care and those who never engage in self-care $(n=5)$.

\section{Self-care and Traumatic Stress $(\mathrm{H} 7)$}

Spearman's Rho correlation coefficients indicated a small significant negative relationship between frequency of self-care and total levels of traumatic stress in all measures of the IES-r except the Avoidance subscale, which had a small nonsignificant negative relationship. IES-Total/mean $\left(r_{\mathrm{s}}=-.249, p=.012\right)$. Mean IESIntrusion ( $\left.r_{\mathrm{s}}=-.225, p=.025\right)$. Mean IES-Avoidance $\left(r_{\mathrm{s}}=-.166, p=.099\right)$. Mean IESHyperarousal $\left(r_{\mathrm{s}}=-.297, p=.003\right)$.

\section{Self-care and Burnout (H7)}

Spearman's Rho correlation coefficients indicated a small significant negative relationship between self-care and burnout in two of Maslach's burnout inventory subscales; Emotional Exhaustion ( $\left.r_{\mathrm{s}}=-.293, p=.003\right)$, Depersonalisation $\left(r_{\mathrm{s}}=-.260\right.$, $p=.009$ ), and a medium positive relationship with Personal Accomplishment subscale $\left(r_{\mathrm{s}}=.392, p<.001\right)$. 
Table 1: Summary of correlational data for inferential statistical analysis

\begin{tabular}{|c|c|c|c|c|c|c|c|}
\hline & $\begin{array}{l}\text { Years } \\
\text { employed }\end{array}$ & $\begin{array}{l}\text { Exposure } \\
\text { to } \\
\text { Violence }\end{array}$ & $\begin{array}{l}\text { Self- } \\
\text { Care }\end{array}$ & $\begin{array}{l}\text { Traumatic } \\
\text { Stress }\end{array}$ & $\begin{array}{l}\text { Emotional } \\
\text { Exhaustion }\end{array}$ & Depersonalisation & $\begin{array}{l}\text { Personal } \\
\text { Accomplishment }\end{array}$ \\
\hline Years employed & 1 & & & & & & \\
\hline $\begin{array}{l}\text { Exposure to } \\
\text { Violence }\end{array}$ & $-.276^{\star *}$ & 1 & & & & & \\
\hline Self-Care & 0.118 & $-.217^{\star}$ & 1 & & & & \\
\hline Traumatic Stress & $-.268^{\star *}$ & $.527^{\star \star}$ & $-.249^{*}$ & 1 & & & \\
\hline $\begin{array}{l}\text { Emotional } \\
\text { Exhaustion }\end{array}$ & -0.027 & $.534^{\star \star}$ & $.293^{* \star}$ & $.497^{\star \star}$ & 1 & & \\
\hline Depersonalisation & 0.017 & $.500^{\star \star}$ & $.260^{\star \star}$ & $.484^{\star *}$ & $.726^{\star \star}$ & 1 & \\
\hline $\begin{array}{l}\text { Personal } \\
\text { Accomplishment }\end{array}$ & 0.096 & $-.476^{\star \star}$ & $.392^{\star \star}$ & $-.419^{\star \star}$ & $-.527^{\star \star}$ & $-.620^{\star \star}$ & 1 \\
\hline
\end{tabular}

\section{Discussion}

\section{Interpretations}

As previously highlighted, the demand for all types of public and private health and social care services in Ireland will increase exponentially by 2030 (Wren et al., 2017). Physical violence and aggression have been described as a characteristic aspect of SCW (Keogh and Byrne, 2016), and this has been linked to increased levels of stress and burnout in staff (Lloyd, King and Chenoweth, 2002). Maslach (2016), also predicted burnout would be related to the desire to leave one's job (Maslach et al., 2016). Furthermore, the Crisis, Concern and Complacency report (2016) highlighted the fact that very little attention has been paid to this situation by policy makers since the first report in 2001 (Keogh et al., 2001; Keogh and Byrne, 2016). As a result of this information, it was important to investigate this area further to better understand the relationship between workplace violence/aggression and well-being in SCWs.

The aim of the current research was primarily to examine the relationship between the frequency of exposure to physical violence/aggression and traumatic stress and burnout in SCWs. Secondary analysis included whether there was a correlation between traumatic stress and burnout and also whether any of the predictor variables were found to have a statistically significant relationship with the criterion variables of traumatic stress and burnout. This research also aimed to elucidate whether the frequency of self-care may have a protective role against traumatic stress and burnout symptomology.

In summary, the findings of this research indicate a statistically significant positive relationship between the frequency of exposure to physical aggression/violence and traumatic stress and burnout measures. Current findings also indicate a significant positive correlation between traumatic stress and burnout. When the frequency of exposure to workplace violence/aggression, traumatic stress, and burnout scores 
were examined in relation to SCWs desire to leave the profession in two-to-three years and/or five years, the findings indicate the presence of a significant positive relationship between the variables. Finally, the current findings indicate the presence of a significant negative correlation between self-care and traumatic stress and burnout. Detailed interpretations of each research hypothesis will now be discussed and followed by a critical evaluation of the present research.

Hypothesis 1 (H1): This research hypothesis was accepted; inferential analysis indicated a significant positive relationship between the frequency of exposure to workplace aggression/violence and levels of traumatic stress. Total average IES-r scores for the daily and weekly exposure groups were above the threshold for indicating potential clinical concern for PTSD (Asukai et al., 2002). The daily exposure group had scores high enough to potentially suppress the immune system function for as much as ten years after the traumatic event (Kawamura, Kim and Asukai, 2001). This finding is supported by the Crisis, Concern and Complacency report (2016) which found that $98 \%$ of SCWs exposed to workplace violence experienced stress (Keogh and Byrne, 2016). Rippon (2000) highlighted how violence is becoming a significant concern for healthcare professionals and that primary, secondary and tertiary victims may experience symptoms of PTSD (Rippon, 2000). The current findings indicate that traumatic stress resulting from workplace violence exposure may be dependent on the frequency of exposure, with higher frequencies resulting in higher levels of traumatic stress.

Hypothesis 2 (H2): This research hypothesis was accepted; Inferential analysis indicated a significant positive relationship between the frequency of exposure to workplace aggression/violence and burnout subscales of emotional exhaustion (EE) and depersonalisation (DP). Analysis also indicated a significant negative relationship between workplace aggression/violence and personal accomplishment (PA). This finding is supported by previous studies examining the role of exposure to violence and burnout in various professions including; Nurses (Galián-Muñoz et al., 2016), nursing home caregivers (Isaksson et al., 2008), police officers (Kop, Euwema, and Schaufeli, 1999), Psychiatrists (Kumar, 2007), and social workers (Beaver, 1999).

Hypothesis 3 (H3): This research hypothesis was accepted; Inferential analysis indicated a significant positive correlation between traumatic-stress and burnout subscales EE and DP, and a significant negative correlation with the PA subscale. This result highlights the risk of burnout when SCWs experience elevated levels of stress and may predict desire to leave SCW (Maslach, 2016). A longitudinal study found stress and burnout subscales showed reciprocal causation and a study of clinical psychologists found a relationship between perfectionism, stress, and burnout (D'Souza, Egan, and Rees, 2011; McManus, Winder, and Gordon, 2002).

Hypothesis 4, 5, and 6 (H4, H5 and H6): This research hypothesis was accepted; inferential analysis indicated a significant positive relationship between; frequency of exposure to workplace aggression/violence, traumatic stress, burnout subscales (EE, DP) and desire to leave SCW. Analysis also identified a significant negative relationship between burnout subscale (PA) and desire to leave. The strength of correlation was strongest when SCWs considered their five-year career outlook. Descriptive analysis found that $43 \%$ of SCWs did not wish to be in the profession in 
five years with the highest proportion of SCWs with a desire to leave experiencing daily $(58 \%)$ and weekly (68\%) exposure to violence/aggression. Interestingly, the mean length of service in the daily, weekly, monthly, yearly exposure to violence groups followed a pattern of decreasing length of service with higher levels of exposure to physical aggression/violence, as outlined in figure 1.

Hypothesis $7(\mathbf{H 7})$ : This research hypothesis was accepted; Inferential analysis indicated a significant negative relationship between frequency of self-care and traumatic stress and burnout subscales EE and DP. Analysis also found a significant positive relationship between frequency of self-care and personal accomplishment. The current research indicated that $67 \%$ of SCWs did not feel available supports were adequate to maintain their physical and mental well-being. As the frequency of selfcare during SCWs personal time has been indicated in the current research to have an ameliorative effect on SCW stress and burnout, it is important for employers to consider policy changes to allow self-care activities during work to reduce stress and burnout in staff. SCW is often characterised by a lack of dedicated lunch breaks and shifts can often be twelve hours in length with consecutive shifts. One policy change employers could make may be to provide additional support staff to allow SCWs to have guaranteed lunch breaks.

\section{Limitations}

Potential limitations may include the small sample size of 100 participants that may not allow for adequate statistical strength and confidence. Results may be more significantly impacted by outliers. The crisis, concern and complacency report by Keogh and Byrne (2016), which has informed this current study had 402 participants. Furthermore, the field of SCW is very diverse and most participants in this study worked in residential services $(n=87)$ which have been characterised as being complex and unpredictable environments (Clough, Bullock and Ward, 2006). Howard (2014) stresses that residential care can be chaotic, ambivalent, turbulent, unpredictable, and often dangerous for staff and young people (Howard, 2014). This may have resulted in the survey population being primarily composed of SCWs exposed to higher frequencies of physical aggression/violence. In addition to sample size, the method of investigation involved the use of self-reported questionnaires which have some flaws. Participants may not always have the necessary selfreflection skills to provide unbiased and accurate self-reporting. Participants may also be fearful of being perceived as unskilled or not up to the job (Keogh et al., 2001). As the survey was completed during working hours, time constraints may have resulted in participants not taking adequate time to fully consider each question and response.

\section{Strengths}

The current research has several attributes which may qualify as strengths. There was a gender balance (56\% female, $44 \%$ male) and wide age range (21-54). Participants had a wide range of experience in SCW ( 6 months -25 years). Both full and part-time staff and front-line and management participated in the study. The questionnaire was completely anonymous which may have given participants more confidence to give honest responses. Questionnaire length was relatively short (5-10 minutes), ensuring participants didn't lose patience or interest and would give each question sufficient attention and thought. This helped to give a $100 \%$ survey completion rate across 
participants. This research may also be unique in that it examined the potential protective role of self-care in moderating the perceived effects of workplace violence/aggression on traumatic stress and burnout in SCWs

The revised Impact of Events Scale and Maslach Burnout Inventory (MBI) are well researched and validated. The reliability of the MBI-HSS exceeds the recommended levels for research instruments and the validity has been demonstrated by numerous studies and meta-analytic reviews confirming hypotheses regarding relationships between job characteristics and burnout (Maslach, 2016). The revised Impact of Event Scale is one of the most widely used self-report measures for traumatic stress and has high reliability and validity scores (Beck et al., 2008).

\section{Future Research}

Future research would benefit from addressing some of the limitations of this current research. For example, a larger sample size incorporating a wider and more diverse sample of SCWs in different settings may give a more accurate representation of the full spectrum of SCW experiences. To counteract weaknesses in self-report questionnaires, future research may also benefit from asking spouses/significant others to answer questions on behalf of SCWs. Future research may also benefit from investigating criterion variables pre-and-post self-care education and engagement.

\section{Implications and Applications}

The current research has implications and applications for social care policy, care providers and in supporting SCWs to ensure optimal well-being is maintained by encouraging self-care activities and by providing additional staff supports to allow rest periods and support during/after incidents of physical violence/aggression. Social care employers may introduce training and awareness of the important benefits of self-care in ameliorating the detrimental impact of stressful events, and in doing so may reduce the potential for staff burnout and desire to leave SCW. As previously discussed, the ESRI found that demand for public and private health and social care services in Ireland will increase exponentially by 2030 (Wren et al., 2017). These changes will have significant and profound impacts on the health and social care sector which is already characterised by challenges of staff retention (Keogh and Byrne, 2016; Barak, Nissly and Levin, 2001). By examining the factors that may lead to staff leaving their job and indeed the SCW profession itself, and also the factors that may alleviate this, there is an opportunity to improve social care policy and care provision. This may improve staff retention and all the benefits this entails, such as: consistency of staff/care, higher quality of trained/experienced staff, leading to higher quality of care provided, lower costs to care providers for recruitment campaigns and sick leave etc.

\section{Conclusion}

This study examined the relationship between frequency of exposure to physical violence/aggression and several variables. The results of this research indicate a statistically significant positive relationship between the frequency of exposure to physical aggression/violence and traumatic stress and burnout measures. Current findings also indicate a significant positive correlation between traumatic stress and burnout. Exposure to workplace violence/aggression, traumatic stress, and burnout 
also positively correlated with SCWs desire to leave the profession in the next five years. Results also highlighted several interesting points, such as $92 \%$ of SCWs believing a culture of normality towards exposure to physical violence/aggression exists. Eighty-one percent of participants also believed their employer expected them to be exposed to workplace violence. The results also indicated the average length of time SCWs stay in the profession is five years. This decreases to three years and four years for SCWs exposed to physical violence daily/weekly respectively. Finally, these findings indicate the presence of a significant protective role of self-care activities in moderating the perceived effects of workplace violence/aggression on traumatic stress and burnout in SCWs. This protective role of self-care may help ameliorate current rates of traumatic stress and burnout in SCWs.

\section{References}

Alink, L. R., Euser, S., Bakermans-Kranenburg, M. J. and van IJzendoorn, M. H. (2014) 'A challenging job: physical and sexual violence towards group workers in youth residential care', Child and Youth Care Forum, 43(2), pp. 243-250. SocINDEX, EBSCOhost [Online]. (Accessed: 12 October 2018).

Asukai, N., Kato, H., Kawamura, N., Kim, Y., Yamamoto, K., Kishimoto, J., Miyake, Y. and Nishizono-Maher, A. (2002) 'Reliability and validity of the Japanese-language version of the impact of event scale-revised (IES-R-J): four studies of different traumatic events', The Journal of Nervous and Mental Disease, 190(3), pp. 175-182. doi: 10.1097/00005053200203000-00006. (Accessed: 23 October 2018).

Balloch, S., Pahl, J., and McLean, J. (1998) 'Working in the social services: job satisfaction, stress and violence'. The British Journal of Social Work, 28(3), pp. 329-350. doi:

https://dx.doi.org/10.1093/oxfordjournals.bjsw.a011343. (Accessed: 30 December 2018).

Barak, M. E.M., Nissly, J. A. and Levin, A. (2001) 'Antecedents to retention and turnover among child welfare, social work, and other human service employees: what can we learn from past research? A review and metanalysis'. Social Service Review, 75(4), pp. 625-661. doi: 10.1086/323166. (Accessed: 25 December 2018).

Beaver, H. (1999) Client violence against professional social workers: frequency, worker characteristics, and impact on worker job satisfaction, burnout, and health. PhD thesis. University of Arkansas. ProQuest Information and Learning. (Accessed: 25 December 2018).

Beck, J. G., Grant, D. M., Read, J. P., Clapp, J. D., Coffey, S. F., Miller, L. M. and Palyo, S. A. (2008) 'The Impact of Event Scale-Revised: Psychometric properties in a sample of motor vehicle accident survivors', Journal of Anxiety Disorders, 22(2), pp. 187-198. doi: 10.1016/j.janxdis.2007.02.007. (Accessed: 12 October 2018).

Brodie, I. (2005) 'Education and residential child care in England: a research perspective', in Crimmens, D. and Milligan, I. (eds.) Facing forward: Residential care in the 21st century. Dorset: Russell House Publishing, pp. 163-171.

Clough, R. Bullock, R. and Ward, A. (2006) What Works in Residential Child Care: A review of research evidence and the practical considerations. London: National Children's Bureau.

Collins, S, and Long, A. (2003) 'Working with the psychological effects of trauma: consequences for mental health-care workers - a literature review', Journal of Psychiatric and Mental Health Nursing, 10(4), pp. 417-424. doi: 10.1046/j.1365-2850.2003.00620.x. (Accessed: 30 December 2018). 
Colton, M. and Roberts, S. (2007) 'Factors that contribute to high turnover among residential child care staff', Child and Family Social Work, 12(2), pp. 133-142. SocINDEX, EBSCOhost [Online]. (Accessed: 12 October 2018).

Colton, M. and Roberts, S. (2006 'The retention of residential group care workers', International Journal of Child and Family Welfare, 9(3), pp. 160-177. (Accessed: 12 October 2018).

Creamer, M., Bell, R. and Failla, S. (2003) 'Psychometric properties of the impact of event scale_revised', Behaviour research and therapy, 41(12), pp. 1489-1496.

https://doi.org/10.1016/j.brat.2003.07.010 (Accessed: 30 December 2018).

D'Souza, F., Egan, S. J. and Rees, C. S. (2011) 'The relationship between perfectionism, stress and burnout in clinical psychologists', Behaviour Change, 28(1), pp. 17-28. Academic Search Complete, EBSCOhost [Online]. (Accessed: 30 December 2018).

Emerson, E. and Hatton, C. (2000) Violence against social care workers supporting people with learning difficulties: a review. Lancaster: University of Lancaster.

Figley C.R. (1995) 'Compassion fatigue as secondary traumatic stress disorder: an overview', in: Figley, C.R. (ed.) Compassion Fatigue: Coping with Secondary Traumatic Stress Disorder in Those Who Treat the Traumatized. New York: Brunner/Mazel, pp. 1-20.

Franz, S., Zeh, A., Schablon, A., Kuhnert, S. and Nienhaus, A. (2010) 'Aggression and violence against health care workers in Germany - a cross sectional retrospective survey'. BMC Health Services Research, 10(1), pp. 51-58. Academic Search Complete, EBSCOhost [Online]. (Accessed: 12 October 2018).

Freudenberger, H. J. (1975) 'The staff burn-out syndrome in alternative institutions', Psychotherapy, 12(1), pp. 73-82. APA PsycArticles, EBSCOhost [Online]. (Accessed: 30 December 2018).

Galián-Muñoz, I., Ruiz-Hernández, J. A., Llor-Esteban, B., and López-García, C. (2016) 'User violence and nursing staff burnout: the modulating role of job satisfaction'. Journal of Interpersonal Violence, 31(2), 302-315. https://doi.org/10.1177/0886260514555367 (Accessed: 27 December 2018).

Harris, B. and Leather, P. (2012) 'Levels and consequences of exposure to service user violence: evidence from a sample of UK social care staff', British Journal of Social Work, 42(5), pp. 851-869. SocINDEX, EBSCOhost [Online]. (Accessed: 12 October 2018).

Hastings, R. P. and Brown, T. (2002) 'Behavior problems of children with autism, parental self-efficacy, and mental health', American Journal on Mental Retardation, 107(3), pp. 222232. doi:10.1352/0895-8017(2002)107<0222:BPOCWA>2.0.CO;2 (Accessed: 19 October 2018).

Health and Safety Authority (2014) Managing the Risk of Work-related Violence and Aggression in Healthcare: Information Sheet. Available at: https://www.hsa.ie/eng/Publications and Forms/Publications/Information Sheets/Managing the Risk of Workrelated Violence and Aggression in Healthcare Information Sheet.html (Accessed: 23 December 2018). 
Healy, K., Meagher, G., and Cullin, J. (2007) 'Retaining novices to become expert child protection practitioners: creating career pathways in direct practice', British Journal of Social Work, 39(2), pp. 299-317. doi:10.1093/bjsw/bcm125. (Accessed: 23 October 2018).

Hensel, J. M., Lunsky, Y. and Dewa, C. S. (2012) 'Exposure to client aggression and burnout among community staff who support adults with intellectual disabilities in Ontario,

Canada', Journal of Intellectual Disability Research, 56(9), pp. 910-915. FRANCIS Archive, EBSCOhost [Online]. (Accessed: 25 December 2018).

Honkonen, T., Ahola, K., Pertovaara, M., Isometsä, E., Kalimo, R., Nykyri, E., Aromaa, A. and Lönnqvist, J. (2006) 'The association between burnout and physical illness in the general population-results from the Finnish Health 2000 Study', Journal of Psychosomatic Research, 61(1), pp. 59-66. doi:10.1016/j.jpsychores.2005.10.002. (Accessed: 30 December 2018).

Howard, N. (2014) 'The Irish association of social care workers', in Howard, D. and Lyons, D. (eds.) Social care: learning from practice. Dublin: Gill and MacMillan, pp. 12-23.

Isaksson, U., Graneheim, U. H., Richter, J., Eisemann, M., and Åström, S. (2008) 'Exposure to violence in relation to personality traits, coping abilities, and burnout among caregivers in nursing homes: a case-control study', Scandinavian Journal of Caring Sciences, 22(4), pp. 551-559. Academic Search Complete, EBSCOhost [Online]. (Accessed: 27 December 2018).

Kawamura, N., Kim, Y., and Asukai, N. (2001) 'Suppression of cellular immunity in men with a past history of posttraumatic stress disorder', American Journal of Psychiatry, 158(3), pp. 484-486. doi:10.1176/appi.ajp.158.3.484. (Accessed: 23 October 2018).

Keogh, P. and Byrne, C. (2016) Crisis, concern and complacency: a study on the extent, impact and management of workplace violence and assault on social care workers. Dublin: Social Care Ireland.

Keogh, P., Fitzpatrick, A., Hopkins, G., Greene, S., Andrews, N., Ryan, R. and Bailey, P. (2001) The Nature and Extent of Workplace Violence Experienced by Social Care Workers: An Irish Report. Dublin: Irish Association of Care Workers.

Kop, N., Euwema, M. and Schaufeli, W. (1999) 'Burnout, job stress and violent behaviour among Dutch police officers', Work and Stress, 13(4), pp. 326-340. Business Source Complete, EBSCOhost [Online]. (Accessed: 27 December 2018).

Kumar, S. (2007) 'Burnout in psychiatrists', World Psychiatry, 6(3), pp.186-189. Available at: https://www.ncbi.nlm.nih.gov/pmc/articles/PMC2175073/\#? (Accessed: 27 December 2018).

Lalor, K. and Share, P. (2013) 'Understanding social care', in: Lalor, K. and Share, P. (eds.) Applied social care: an introduction for students in Ireland. 3rd edn. Dublin: Gill and Macmillan, pp. 3-18.

Littlechild, B. (2000) 'I know where you live': how child protection social workers are affected by threats and aggression. A study into the stresses faced by child protection workers in Hertfordshire. Hatfield: University of Hertfordshire.

Lloyd, C., King, R. and Chenoweth, L. (2002) 'Social work, stress and burnout: a review', Journal of Mental Health, 11(3), pp. 255-265. FRANCIS Archive, EBSCOhost [Online]. (Accessed: 21 March 2019). 
Lovell, A. and Skellern, J. (2013) 'Tolerating violence: a qualitative study into the experience of professionals working within one UK learning disability service'. Journal of Clinical Nursing, 22(15-16), 2264-2272. doi: 10.1111/jocn.12164. (Accessed: 25 December 2018).

Lundström, M., Åström, S. and Graneheim, U. H. (2007) 'Caregivers' experiences of exposure to violence in services for people with learning disabilities', Journal of Psychiatric and Mental Health Nursing, 14(4), 338-345. doi:10.1111/j.1365-2850.2007.01081.x. (Accessed: 25 December 2018).

Maslach, C., Jackson, S. E., Leiter, M. P., Schaufeli, W. B. and Schwab, R. L. (2016) Maslach burnout inventory manual. $4^{\text {th }}$ edn. Menlo Park CA: Mind Garden, Inc.

McAdams, C. R. (2002) 'Trends in the occurrence of reactive and proactive aggression among children and adolescents: Implications for preparation and practice in child and youth care', Child and Youth Care Forum, 31(2), pp. 89-109. SocINDEX, EBSCOhost [Online]. (Accessed: 12 October 2018).

McKenna, K. (2004) Study of work-related violence. Committee on Workplace Violence, North Eastern Health Board, pp. 1-121.

McManus, I. C., Winder, B. C. and Gordon, D. (2002) 'The causal links between stress and burnout in a longitudinal study of UK doctors', The Lancet, 359(9323), pp. 2089-2090. Business Source Complete, EBSCOhost [Online]. (Accessed: 11 February 2019).

Office for National Statistics (2017) 'Suicide by Occupation, England: 2011 to 2015'. Available at:

https://www.ons.gov.uk/peoplepopulationandcommunity/birthsdeathsandmarriages/deaths/ar ticles/suicidebyoccupation/england2011to2015 (Accessed: 25 December 2018).

Rippon, T. J. (2000) 'Aggression and violence in health care professions', Journal of Advanced Nursing, 31(2), pp. 452-460. doi: 10.1046/j.1365-2648.2000.01284.x. (Accessed: 25 December 2018).

Ruotsalainen, J. H., Verbeek, J. H., Mariné, A., and Serra, C. (2016) 'Preventing occupational stress in healthcare workers', Sao Paulo Medical Journal, 134(1), p. 92. https://doi.org/10.1590/1516-3180.20161341T1. (Accessed: 23 October 2018).

Santos, A., Leather, P., Dunn, J., and Zarola, A. (2009) 'Gender differences in exposure to co-worker and public-initiated violence: assessing the impact of work-related violence and aggression in police work', Work and Stress, 23(2), pp. 137-154. Business Source Complete, EBSCOhost [Online]. (Accessed: 30 December 2018).

Schat, A. C. and Kelloway, E. K. (2000) 'Effects of perceived control on the outcomes of workplace aggression and violence', Journal of Occupational Health Psychology, 5(3), pp. 386-402. APA PsycArticles, EBSCOhost [Online]. (Accessed: 27 December 2018).

Smith, M., and Nursten, J. (1998) 'Social workers' experience of distress - moving towards change?', The British Journal of Social Work, 28(3), pp. 351-368. JSTOR Journals, EBSCOhost [Online]. (Accessed: 27 December 2018).

Spitzer, R. L. and Williams, J. B. (1980) DSM III: Diagnostic and Statistical Manual of Mental Disorders. $3^{\text {rd }}$ edn. Washington, DC: American Psychiatric Association.

Taylor, M. (2011) 'Understanding and responding to the emotional challenge of working in residential child care settings: the case for staff support groups', European Journal of Social 
Education, (20/21), pp. 64-80. SocINDEX, EBSCOhost [Online]. (Accessed: 25 December 2018).

Weiss, D. S. and Marmar, C. R. (1997) 'The impact of event scale-revised', in Wilson, J.P. and Keane, T.M. (eds.) Assessing Psychological Trauma and PTSD: a Practitioner's Handbook. New York: Guilford Press, pp. 399-411.

Whitaker, D., Archer, L., and Hicks, L. (1998) Working in Children's Homes: Challenges and Complexities. London: Wiley.

World Health Organization (2009) Reducing Violence through Victim Identification, Care and Support Programmes. Available at:

https://www.who.int/violence injury prevention/violence/programmes.pdf. (Accessed: 27 December 2018).

Wren, M. A., Keegan, C., Walsh, B. M., Bergin, A., Eighan, J., Brick, A., Connolly, S., Watson, D. and Banks, J. (2017) Projections of Demand for Healthcare in Ireland, 20152030: First Report from the Hippocrates Model. Dublin: Economic and Social Research Institute. 